\title{
Unassisted aircraft landing via co-operative data exchange
}

\author{
Vijay Ragothaman*, Fariha Baloch, Ravi Pendse \\ Department of Electrical \& Computer Engineering, College of Engineering
}

\begin{abstract}
The Air Traffic Controller (ATC) is required to maintain a safe and orderly flow of airplanes in any airport. A fairly recent incident at the Los Angeles Airport (LAX) caused a blackout to the entire control tower and resulted in a number of aircrafts being stranded in the air, due to the lack of communication with the ATC. This incident has caused widespread concern in the aircraft community and needs to be addressed so that future occurrences can be avoided.
\end{abstract}

\section{Introduction}

Automatic Dependent Surveillance - Broadcast (ADS-B) is a Global Positioning System (GPS) based air-traffic surveillance technology. The concept behind ADS-B is that an aircraft can automatically broadcast its identification address, GPS derived latitude and longitude, altitude, and the 3-D velocity. This broadcast information from ADS-B can be picked up by the ATC and used to track aircrafts more precisely than with radar. This concept can be extended to involve other aircrafts in the same airspace to be equipped with an ADS-B receiver, hence allowing them to listen to these broadcasts and be able to know the location of others aircrafts.

The system proposed in this paper is intended to serve as a supplementary mechanism to existing air-ground systems and can be extended to operate on its own in case of unforeseen circumstances such as loss of power to the ATC.

\section{ADS-B}

The way ADS-B works can be explained with a simple example by considering the operation of ATC system radar. This radar measures the bearing and range of an aircraft. The aircraft bearing is determined when the radar receives a reply to its interrogation from the aircraft and its range by the time taken to receive the reply from the aircraft. As the aircraft gets farther, the beam of the antenna becomes wider making the measured information less accurate. In ADS-B systems the position of the aircraft is determined from its broadcast reports. These reports are accurate because they are determined by accurate position systems such as GPS

\section{IP Connectivity}

In [1] and [2], the authors have shown that the available IP connectivity between aircraft and ground can be used as a supplementary mechanism to aid communication and navigation from aircraft to ground. This connectivity is established with the help of satellite links and Mobile IP [3] and the concept can be extended to provide support for surveillance related information.

Ad-Hoc Networks

An Ad-hoc network is an infrastructure-less network created on the fly, based on need. The function of nodes in such a network is not limited to sending and receiving data, but also in forwarding packets meant for other nodes. This means that nodes function as hosts as well as routers. Wireless media is used to link these nodes together. These networks are self-organizing networks and can work in a stand-alone mode or in conjunction with wired networks. The concept of ad-hoc networking can be extended and applied to the current scenario in ADNs.

\section{Co-operative Data Exchange}

This section describes the ability to use the available IP connectivity to serve as a supplementary mechanism to existing ADS-B systems. In worst-case scenarios such as loss of power to the ATC, this system can bring about the possibility of landing an aircraft without assistance from the ground. 
Co-operative Data Exchange (CDE) refers to the exchange of information between aircrafts in flight. The authors present an algorithm which serves as a set of guidelines for $\mathrm{CDE}$ using the concept of Ad-Hoc networks.

\section{Algorithm}

- When loss of ATC is detected, the pilot switches over to CDE. The aircraft broadcasts a 'CDE-JOIN' message.

- Aircrafts which receive these messages start populating a database with this information and compute ' $p$ ' for corresponding aircrafts. ' $p$ ' is defined as the safe distance of separation between aircrafts to avoid collisions.

- Each aircraft compares its Critical Code (CC) with the value in the CDE-JOIN packet. If its own code is lower, then it will just pass the packet on to the next neighbor. In the other case, where its code is higher, it will discard the packet and send out one on its own.

- All aircrafts which are within 'd' distance of each other will update their databases with all this information and the order in which they can land is determined.

- Once the database has been updated with the order of landing, the aircrafts will calculate the time it will take for all aircrafts need to land. For example, if the first aircraft's landing process ( enabling landing gear etc) takes $\mathrm{T}$ minutes, then to land $\mathrm{N}$ aircrafts it will take $\mathrm{N} * \mathrm{~T}$ minutes, where $\mathrm{N}$ is the number of entries in the database of the aircrafts.

- For next $\mathrm{N}^{*} \mathrm{~T}$ minutes, these aircrafts that have formed an ad-hoc network will not accept other entries. If new aircraft or aircrafts comes within a distance ' $\mathrm{d}$ ' while the landings are taking place than, they will from there own ad-hoc network and start the process of determining the landing schedule.

- To avoid collision between two aircrafts of different ad-hoc networks, it is crucial that the second set of aircrafts know $\mathrm{N}^{*} \mathrm{~T}$ time, i.e. the end of the landing times for first set.

- Hence, the last aircraft in the database of the first ad-hoc network will advertise this time by sending a TIME_END message. This message will contain the ad-hoc network's ID and the time $\mathrm{N} *$ T minutes.

- Upon reception if this message the new ad-hoc network set will start landing process at $(\mathrm{N} * \mathrm{~T})+1$ minutes.

- The process will be repeated and will continue until ATC becomes available. Notifications can be made via CPDLC as outlined in [2].

\section{Conclusions}

The system presented here can also be used to co-exist with current ADS-B implementations. In case of ADS-B failure, this system can run independently and communicate to ground stations via the IP link to safely land aircrafts in worst-case scenarios. Listed below is some of the work that needs to be carried out.

- Development of a model which will more accurately reflect the algorithm.

- Simulation of such a scenario to prove its merit.

- Detailed and accurate consideration of all parameters which are critical to safe landing of an aircraft.

\section{References}

[1] Ragothaman, Thanthry, Bhagavathula and Pendse, 2004, IP Connectivity and DAP, Wichita State University, Digital Avionics Systems Conference.

[2] Ragothaman, Baloch and Pendse, 2005, Transport of Flight Critical Data over Internet Protocol, Wichita State University, Digital Avionics Systems Conference

[3] Perkins, 1998, Mobile Networking through Mobile IP, IEEE Internet Computing. 